\section{Transcriptome Profiling Data of Botrytis cinerea Infection on Whole Plant Solanum lycopersicum}

\author{
Dhruv Aditya Srivastava, ${ }^{1}$ Gulab Chand Arya, ${ }^{1}$ Eswari PJ Pandaranayaka, ${ }^{1}$ Ekaterina \\ Manasherova, ${ }^{1}$ Dov B. Prusky, ${ }^{2}$ Yigal Elad, ${ }^{3}$ Omer Frenkel, ${ }^{3}$ and Arye Harel ${ }^{1, \dagger}$ \\ ${ }^{1}$ Department of Vegetable Research, Institute of Plant Sciences, Agricultural Research Organization, \\ Volcani Center, 68 HaMaccabim Road, P.O. Box 15159, Rishon LeZion 7505101, Israel \\ ${ }^{2}$ Department of Postharvest Science, Institute of Postharvest and Food Sciences, Agricultural Research \\ Organization, Volcani Center, 68 HaMaccabim Road, P.O. Box 15159, Rishon LeZion 7505101, Israel \\ ${ }^{3}$ Department of Plant Pathology and Weed Research, Institute of Plant Protection, Agricultural Research \\ Organization, Volcani Center, 68 HaMaccabim Road, P.O. Box 15159, Rishon LeZion 7505101, Israel
}

\begin{abstract}
Botrytis cinerea is a foliar necrotrophic fungal-pathogen capable of infecting $>580$ genera of plants, is often used as model organism for studying fungal-host interactions. We used RNAseq to study transcriptome of $B$. cinerea infection on a major (worldwide) vegetable crop, tomato (Solanum lycopersicum). Most previous works explored only few infection stages, using RNA extracted from entire leaf-organ diluting the expression of studied infected region. Many studied B. cinerea infection, on detached organs assuming that similar defense/ physiological reactions occurs in the intact plant. We analyzed transcriptome of the pathogen and host in 5 infection stages of whole-plant leaves at the infection site. We supply high quality, pathogen-enriched gene count that facilitates future research of the molecular processes regulating the infection process.
\end{abstract}

\section{Transcriptome Announcement}

Botrytis cinerea is an important pathogen that is commonly used as a model of a necrotrophic fungal phytopathogen (Dean et al. 2012; Williamson et al. 2007). B. cinerea, the causal agent of gray mold disease, is capable of infecting over 580 genera, including abundantly used crops such as tomatoes, grapevines, and berries (Dean et al. 2012; Elad et al. 2016). The infection affects foliar organs of the plant, causing severe symptoms, such as soft rot, lesions, and fruit drop, ultimately leading to host death. According to the United Nations Food and Agriculture Organization, tomato, Solanum lycopersicum, is the second major crops in the world. Gray mold disease is abundant in greenhouse tomato plants, due to optimal conditions of high humidity and foliar density (Stall 1991) and to susceptibility to stem infection that often follows the pruning of leaves and side shoots.

Obtaining transcription profiles of B. cinerea infection in S. lycopersicum (cv. M82) is important for a number of reasons. First, transcriptomics was shown to be a major research tool used to understand dynamics of underlying molecular changes supporting biotic interactions during the infection process. However, many interesting studies of $B$. cinerea

${ }^{\dagger}$ Corresponding author: A. Harel; aryeharel@ volcani.agri.gov.il

*The $e$-Xtra logo stands for "electronic extra" and indicates there are supplementary materials published online.

The author(s) declare no conflict of interest.

Accepted for publication 16 June 2020.

Modified: 8 Oct 2021.

(c) 2020 The American Phytopathological Society
$e-\mathbf{X t r a}^{*}$

Funding

This work was supported by Israeli Chief Scientist, Ministry of Agriculture grant 20-10-0069 and an ARO postdoctoral fellowship grant 19/2020.

\section{Keywords}

Botrytis cinerea, fungus-plant interaction, plant response to pathogen, Solanum lycopersicum, transcriptome 
infection process were directed only to the analysis of the plant-host transcriptome in Arabidopsis (Windram et al. 2012), tomato (Vega et al. 2015), tomato fruit (Blanco-Ulate et al. 2013), and strawberry fruits (Haile et al. 2019). Other studies were focused on transcript dynamics involved in the plants with limited analysis of the $B$. cinerea transcriptome. The transcriptome of $B$. cinerea infection was previously studied in various hosts such as i) lettuce (De Cremer et al. 2013), with less than 80,000 reads mapped to each $B$. cinerea sample of early infection (12 and $24 \mathrm{~h}$ postinfection [hpi]); ii) wild tomato (Smith et al. 2014), detached leaves with less than 10,000 reads mapped to $B$. cinerea in each of the infection stages; iii) grapevine fruits (Haile et al. 2020; Kelloniemi et al. 2015); and iv) cucumber at the late infection stage (96 hpi) (Kong et al. 2015). Few exceptions that also used high read counts to study the $B$. cinerea transcriptome were performed in fruits of grapevine as opposed to leaf tissue in our work (Haile et al. 2020) and in late infection stage (96 hpi) of cucumber infection, which is part of family Cucurbitaceae (Kong et al. 2015), as opposed to four infection stages (+0 hpi) in the Solanaceae representative used in our study. Other interesting studies have used high-depth sequencing from detached leaf tissue to explore genetic variability in transcriptomes of $96 \mathrm{~B}$. cinerea isolates at $16 \mathrm{hpi}$ of the Arabidopsis wild-type (Col-0) (Soltis et al. 2020) and two mutants with jasmonate- or salicylic acid-compromised immunities (Zhang et al. 2019). The authors acknowledge that, although sequence depth is critical to obtain measurements on numbers of genes, at a certain depth, there is a rapid loss of new gene information per unit read, and it is better to use more samples to obtain better statistical power, as done in this work by using three samples per treatment.

Secondly, previous study of $B$. cinerea infection on tomato has illustrated that inhibition of host photosynthetic electron transport was restricted to the direct vicinity of the infection site (Berger et al. 2004). This work illustrates the importance of measuring transcription at the infections site as opposed to extraction of RNA from entire leave tissue, which would dilute expression of interest related to infection by non-infected background. However, distal host responses to $B$. cinerea infection (e.g., in the infected organ or in systemic acquired resistance) could play an important role in plant fungal interactions. Therefore, for studies that are aimed to gain more holistic understanding of plant defense mechanisms, it is also important to explore transcriptome in remote regions.

Third, the danger posed by fungal pathogens is enhanced by accelerated evolution of pathogens mainly due to continued utilization of fungicides in monoculture practice, human or climate-dependent dispersal, and their "genomic plasticity" enabling formation of genetic variations in these phytopathogens, which allows them to adapt quickly to human systems (Giraldo and Valent 2013; Niño-Sánchez et al. 2015; Raffaele and Kamoun 2012; Srivastava et al. 2018). In B. cinerea, this process is reflected by the emergence of isolates that are resistant to several classes of fungicides in multiple geographical locations (Hahn 2014; Rupp et al. 2017) and even by the emergence of multiple fungicide-resistant strains (Elad et al. 2016; Rupp et al. 2017). Improving our understanding of the infection process using advanced genomics and transcriptomics technologies (Pandaranayaka et al. 2019) should facilitate future development of new and existing strategies that will enable us to control plant pathogens.

In this study, we supply high-quality data of five infection stages of $B$. cinerea B05.10 (van Kan et al. 1997) on S. lycopersicum (cv. M82); in each stage, three biological replicates were used. The B05.10 strain used throughout this study was routinely cultured on potato dextrose agar (Difco) adjusted to $2 \%$ agar with $0.25 \mathrm{wt} / \mathrm{vol}$ chloramphenicol (Sigma) at $18^{\circ} \mathrm{C}$ in dark, unless otherwise specified. Tomato seedlings were routinely grown in green house at $25^{\circ} \mathrm{C}$ for four to five weeks (under 16-h fluorescent-based light and 8-h dark regimes in Green quality soil mix, Tuff soil [Merom Golan]) before inoculation of whole plants. Plants were transferred to 2-liter plastic pots in a growth chamber controlled at $22 \pm 1^{\circ} \mathrm{C}$ with $16-\mathrm{h}$ fluorescent-based light and 8-h dark regimes and were acclimatized for 1 week before inoculation. The adaxial sides of tomato plant leaves were inoculated with 1,000 spores of $B$. cinerea (six inoculation sites per leaf, four leaves per plant), were suspended in $5 \mu$ l of onefourth potato dextrose broth (Difco) to facilitate better synchronization of spore germination and to achieve infection of $100 \%$ of the inoculated sites (Elad et al. 1994; Srivastava et al. 2018). Disease progression was monitored at 0, 16, 23, 40, and $47 \mathrm{hpi}$; 23- and 47-hpi measures were taken under red light $1 \mathrm{~h}$ before light was turned on. Minor necrotic patches started to appear only at $23 \mathrm{hpi}$, and establishment of necrotic growth was observed at $40 \mathrm{hpi}$. Note that the plant responds to the red light via the phytochrome system, while $B$. cinerea

1104 / Molecular Plant-Microbe Interactions 
Table 1. Transcriptome statistics of Botrytis cinerea (B05.10) and tomato (Solanum lycopersicum cv. M82) during infection

\begin{tabular}{|c|c|c|c|c|c|}
\hline \multirow[b]{2}{*}{ Parameters } & \multicolumn{5}{|c|}{ Timepoint (hours postinfection) } \\
\hline & 0 & 16 & 23 & 40 & 47 \\
\hline Total read ${ }^{\mathrm{a}}$ & $71,437,565$ & $135,776,539$ & $146,040,697$ & $73,059,701$ & $62,568,364$ \\
\hline \multicolumn{6}{|c|}{ Botrytis cinerea (B05.10) } \\
\hline Mapped reads ${ }^{\mathrm{a}, \mathrm{b}}$ & 2,404,324 (582,037 minimal sample) & $6,265,170$ & $29,849,404$ & $35,571,601$ & $23,589,882$ \\
\hline Mapped CDS ${ }^{\mathrm{C}}$ & 10,496 & 9,870 & 10,844 & 11,096 & 10,916 \\
\hline $\operatorname{Ref} \operatorname{CDS}(\%)^{d}$ & $87.39 \%$ & $82.18 \%$ & $90.29 \%$ & $92.39 \%$ & $90.89 \%$ \\
\hline High Count $\mathrm{CDS}^{\mathrm{e}}$ & 8,174 & 7,671 & 9,288 & 9,647 & 9,352 \\
\hline Ref high CDS (\%) & $68.06 \%$ & $63.87 \%$ & $77.34 \%$ & $80.32 \%$ & $77.87 \%$ \\
\hline \multicolumn{6}{|c|}{ Solanum lycopersicum (cv. M82) } \\
\hline Mapped reads ${ }^{\mathrm{b}}$ & $49,344,680(7,741,620$ minimal sample $)$ & $93,327,812$ & $77,880,059$ & $24,234,150$ & $22,679,766$ \\
\hline Mapped CDS & 22,797 & 24,665 & 24,259 & 22,543 & 22,213 \\
\hline Ref CDS $(\%)^{d}$ & $65.36 \%$ & $70.72 \%$ & $69.55 \%$ & $64.63 \%$ & $63.69 \%$ \\
\hline High Count CDS ${ }^{e}$ & 18,753 & 21,435 & 20,670 & 17,840 & 17,225 \\
\hline Ref high CDS (\%) & $53.77 \%$ & $61.46 \%$ & $59.26 \%$ & $51.15 \%$ & $49.39 \%$ \\
\hline
\end{tabular}

${ }^{a}$ Results represent sum of three replicates unless otherwise specified.

b Reads that were finally mapped to fungi or tomato.

c Number of coding sequences (CDS) having a nonzero count for at least one biological replicate.

d Percent of CDSs in reference genomes of $B$. cinerea and $S$. lycopersicum.

e Mapping based only for genes having an average count of 10 or more for at least one biological replicate.

genome contains receptors that respond to both red and green light (the latter is often used for sampling of plants in the dark) (Schumacher 2017). However, red light was shown to repress conidiation while green light inhibits conidial germination and mycelial growth, which are pivotal for the infection process of B. cinerea (Schumacher 2017; Zhu et al. 2013). Therefore, to obtain minimal effect on fungal virulence, we have used red light in the sampling procedure. Each biological sample consisted of a pool of three independent plants (i.e., separate plants were used for each timepoint), four leaves per plant (i.e., 12 leaves, inoculated altogether with 72 spots). For $0 \mathrm{hpi}$, RNA was extracted from whole leaf with mock infection (infection medium) immediately taken for extraction and mixed with 20\% (by weight) RNA extracted from spores of $B$. cinerea.

RNA extraction was done following manufacturer instructions (Norgen), followed by DNase treatment (Qiagen). Total RNA extractions were tested for quality using Nanodrop and, subsequently, with Bioanalyzer (Agilent Technologies). RNA sample having a RNA integrity number $>4.1,260 / 280$ ratio $>1.8$, and $260 / 230$ ratio $>1.9$ were used to prepare libraries. Libraries were prepared at the Crown Genomics institute of the Nancy and Stephen Grand Israel National Center for Personalized Medicine, Weizmann Institute of Science. Total RNA (500 ng for each sample) was processed using the in-house poly A-based RNA-seq protocol. Libraries were evaluated by Qubit and TapeStation. Samples were sequenced on four lanes of Illumina NextSeq machine, using the Single-Read 75 protocol. The output was, on average, 32.5 million reads per sample. Altogether, transcriptome of 15 biological samples was studied, i.e., three biological replicates for each infection stage.

Analysis of fungal expression in the course of infection could be hampered by high enrichment of plant host RNA, which is even higher in early stages of infection that hardly contain visible hyphae. Thus, to allow the analysis of fungal expression, earlier infection stages (up to 16 and $23 \mathrm{hpi}$ ) were sequenced with high depth (approximately 46 million reads per sample) and later infection stages with lower, although still high, depth (approximately 23 million reads per sample). Reads were trimmed using cutadapt (Martin 2011) and were mapped to the B. cinerea B05.10 genome (Ensembl id ASM83294v1 [Van Kan et al. 2017]) and S. lycopersicum genomes assemblies (ITAG3.1 Solanaceae Genomics Network), using STAR (Dobin et al. 2013) v2.4.2a (using the End To End option and out Filter Mismatch NoverL max set to 0.04 ). Approximately $97 \%$ of the uniquely mapped reads were counted, using Htseq-count (Anders et al. 2015) (intersection-strict or union mode for fungi or tomato, respectively). Approximately 23 million reads per sample were mapped to tomato and 6.7 million reads per sample for fungi (average for all 15 samples in each organism). Minimum reads that were mapped per sample within each genome were $7,741,620$, and 582,037 for tomato and fungi, respectively. Hierarchical clustering based on reads per kilobase million values enabled separating all treatments of tomato and fungi. Multidimensional scaling (MDS) 
(using limma, R package [Ritchie et al. 2015]) enabled the separation of treatments of plant and fungal transcriptomes.

Illustrating expression of more than $63 \%$ of the fungal coding sequences in early infection stages (Table 1), this study was able to answer an important challenge of $B$. cinerea transcriptome during infection, showing a major increase compared with most other studies (excepting those of Haile et al. [2020] that worked on grapevine fruits and Kong et al. [2015] who worked on the single late infection stage [96 hpi] of cucumber). This study will shed light on both pathogen infection and defense processes. The presented fungal transcriptome will help plant pathologists to design experiments to study fungal genes required for infection. The transcriptome sequence data in this study was deposited in the National Center for Biotechnology Information (NCBI) Sequence Read Archive under NCBI Biofroject PRJNA628162. The real-time reverse transcription-PCR validation and MDS of samples are available in the Supplementary Materials file.

\section{Author-Recommended Internet Resources}

NCBI BioProject PRJNA628162: https://www.ncbi.nlm.nih.gov/bioproject/? term=prjna628162

Solanaceae Genomics Network: http://solgenomics.net

\section{Literature Cited}

Anders, S., Pyl, P. T., and Huber, W. 2015. HTSeq-A Python framework to work with high-throughput sequencing data. Bioinformatics 31:166-169.

Berger, S., Papadopoulos, M., Schreiber, U., Kaiser, W., and Roitsch, T. 2004. Complex regulation of gene expression, photosynthesis and sugar levels by pathogen infection in tomato. Physiol. Plant. 122:419-428.

Blanco-Ulate, B., Vincenti, E., Powell, A. L. T., and Cantu, D. 2013. Tomato transcriptome and mutant analyses suggest a role for plant stress hormones in the interaction between fruit and Botrytis cinerea. Front. Plant Sci. 4:142.

De Cremer, K., Mathys, J., Vos, C., Froenicke, L., Michelmore, R. W., Cammue, B. P. A., and De Coninck, B. 2013. RNAseq-based transcriptome analysis of Lactuca sativa infected by the fungal necrotroph Botrytis cinerea. Plant Cell Environ. 36: 1992-2007.

Dean, R., Van Kan, J. A. L., Pretorius, Z. A., Hammond-Kosack, K. E., Di Pietro, A., Spanu, P. D., Rudd, J. J., Dickman, M., Kahmann, R., Ellis, J., and Foster, G. D. 2012. The top 10 fungal pathogens in molecular plant pathology. Mol. Plant Pathol. 13:414-430.

Dobin, A., Davis, C. A., Schlesinger, F., Drenkow, J., Zaleski, C., Jha, S., Batut, P., Chaisson, M., and Gingeras, T. R. 2013. STAR: Ultrafast universal RNA-seq aligner. Bioinformatics 29:15-21.

Elad, Y., Köhl, J., and Fokkema, N. J. 1994. Control of infection and sporulation of Botrytis cinerea on bean and tomato by saprophytic bacteria and fungi. Eur. J. Plant Pathol. 100:315-336.

Elad, Y., Vivier, M., and Fillinger, S. 2016. Botrytis, the good, the bad and the ugly. Pages 1-15 in: Botrytis-The Fungus, the Pathogen and its Management in Agricultural Systems. Springer International Publishing, Cham, Switzerland.

Giraldo, M. C., and Valent, B. 2013. Filamentous plant pathogen effectors in action. Nat. Rev. Microbiol. 11:800-814.

Hahn, M. 2014. The rising threat of fungicide resistance in plant pathogenic fungi: Botrytis as a case study. J. Chem. Biol. 7:133-141.

Haile, Z. M., Malacarne, G., Pilati, S., Sonego, P., Moretto, M., Masuero, D., Vrhovsek, U., Engelen, K., Baraldi, E., and Moser, C. 2020. Dual transcriptome and metabolic analysis of Vitis vinifera cv. Pinot Noir berry and Botrytis cinerea during quiescence and egressed infection. Front. Plant Sci. 10:1704.

Haile, Z. M., Nagpala-De Guzman, E. G., Moretto, M., Sonego, P., Engelen, K., Zoli, L., Moser, C., and Baraldi, E. 2019. Transcriptome profiles of strawberry (Fragaria vesca) fruit interacting with Botrytis cinerea at different ripening stages. Front. Plant Sci. 10:1131.

Kelloniemi, J., Trouvelot, S., Héloir, M.-C., Simon, A., Dalmais, B., Frettinger, P., Cimerman, A., Fermaud, M., Roudet, J., Baulande, S., Bruel, C., Choquer, M., Couvelard, L., Duthieuw, N., Ferrarini, A., Flors, V., Le Pêcheur, P., Loisel, E., Morgant, G., Poussereau, N., Pradier, J.-M., Rascle, C., Trdá, L., Poinssot, B., and Viaud, M. 2015. Analysis of the molecular dialogue between gray mold (Botrytis cinerea) and grapevine (Vitis vinifera) reveals a clear shift in defense mechanisms during berry ripening. Mol. Plant-Microbe Interact. 28: 1167-1180.

Kong, W., Chen, N., Liu, T., Zhu, J., Wang, J., He, X., and Jin, Y. 2015. Large-scale transcriptome analysis of cucumber and Botrytis cinerea during infection. PLoS One 10:e0142221.

Martin, M. 2011. Cutadapt removes adapter sequences from high-throughput sequencing reads. EMBnet. J. 17:10.

Niño-Sánchez, J., Tello, V., Casado-Del Castillo, V., Thon, M. R., Benito, E. P., and Díaz-Mínguez, J. M. 2015. Gene expression patterns and dynamics of the colonization of common bean (Phaseolus vulgaris L.) by highly virulent and weakly virulent strains of Fusarium oxysporum. Front. Microbiol. 6:234.

Pandaranayaka, E. P. J., Frenkel, O., Elad, Y., Prusky, D., and Harel, A. 2019. Network analysis exposes core functions in major lifestyles of fungal and oomycete plant pathogens. BMC Genomics 20:1020.

Raffaele, S., and Kamoun, S. 2012. Genome evolution in filamentous plant pathogens: why bigger can be better. Nat. Rev. Microbiol. 10:417-430.

Ritchie, M. E., Phipson, B., Wu, D., Hu, Y., Law, C. W., Shi, W., and Smyth, G. K. 2015. limma powers differential expression analyses for RNA-sequencing and microarray studies. Nucleic Acids Res. 43:e47.

Rupp, S., Weber, R. W. S., Rieger, D., Detzel, P., and Hahn, M. 2017. Spread of Botrytis cinerea strains with multiple fungicide resistance in German horticulture. Front. Microbiol. 7:2075.

Schumacher, J. 2017. How light affects the life of Botrytis. Fungal Genet. Biol. 106: 26-41.

Smith, J. E., Mengesha, B., Tang, H., Mengiste, T., and Bluhm, B. H. 2014. Resistance to Botrytis cinerea in Solanum lycopersicoides involves widespread transcriptional reprogramming. BMC Genomics 15:334.

Soltis, N. E., Caseys, C., Zhang, W., Corwin, J. A., Atwell, S., and Kliebenstein, D. J. 2020. Pathogen genetic control of transcriptome variation in the Arabidopsis thaliana-Botrytis cinerea pathosystem. Genetics 215:303070.2020.

Srivastava, D. A., Yakubov, M., Feldbaum, R., Tish, N., Shoyhet, H., Manasherova, E., et al. 2018. Multiparametric analysis of diversity in Botrytis cinerea isolates from Israel. Phytoparasitica 46:569-581.

Stall, R. E. 1991. Gray mold. Pages 16-17: in: Compendium of Tomato Diseases. J. B. Jones, J. P. Jones, R. E. Stall, and T. A. Zitter, eds. American Phytopathological Society, St. Paul, MN, U.S.A.

van Kan, J. A., van't Klooster, J. W., Wagemakers, C. A., Dees, D. C., and van der Vlugt-Bergmans, C. J. 1997. Cutinase A of Botrytis cinerea is expressed, but not essential, during penetration of gerbera and tomato. Mol. Plant-Microbe Interact 10:30-38. 
Van Kan, J. A. L., Stassen, J. H. M., Mosbach, A., Van Der Lee, T. A. J., Faino, L., Farmer, A. D., Papasotiriou, D. G., Zhou, S., Seidl, M. F., Cottam, E., Edel, D., Hahn, M., Schwartz, D. C., Dietrich, R. A., Widdison, S., and Scalliet, G. 2017. A gapless genome sequence of the fungus Botrytis cinerea. Mol. Plant Pathol. 18:75-89.

Vega, A., Canessa, P., Hoppe, G., Retamal, I., Moyano, T. C., Canales, J., Gutiérrez, R. A., and Rubilar, J. 2015. Transcriptome analysis reveals regulatory networks underlying differential susceptibility to Botrytis cinerea in response to nitrogen availability in Solanum lycopersicum. Front. Plant Sci. 6:911.

Williamson, B., Tudzynski, B., Tudzynski, P., and van Kan, J. A. L. 2007. Botrytis cinerea: the cause of grey mould disease. Mol. Plant Pathol. 8:561-580.

Windram, O., Madhou, P., McHattie, S., Hill, C., Hickman, R., Cooke, E., Jenkins, D. J., Penfold, C. A., Baxter, L., Breeze, E., Kiddle, S. J., Rhodes, J., Atwell, S.,
Kliebenstein, D. J., Kim, Y. S., Stegle, O., Borgwardt, K., Zhang, C., Tabrett, A., Legaie, R., Moore, J., Finkenstadt, B., Wild, D. L., Mead, A., Rand, D., Beynon, J., Ott, S., Buchanan-Wollaston, V., and Denby, K. J. 2012. Arabidopsis defense against Botrytis cinerea: chronology and regulation deciphered by high-resolution temporal transcriptomic analysis. Plant Cell 24:3530-3557.

Zhang, W., Corwin, J. A., Copeland, D. H., Feusier, J., Eshbaugh, R., Cook, D. E., Atwell, S., and Kliebenstein, D. J. 2019. Plant-necrotroph co-transcriptome networks illuminate a metabolic battlefield. eLife 8:e44279.

Zhu, P., Zhang, C., Xiao, H., Wang, Y., Toyoda, H., and Xu, L. 2013. Exploitable regulatory effects of light on growth and development of Botrytis cinerea. J. Plant Pathol. 95:509-517.

This article was modified on 8 Oct 2021. 


\section{ERRATUM / Volume 33, Number 9, 2020 / MPMI-05-20-0109-A}

In the article "Transcriptome Profiling Data of Botrytis cinerea Infection on Whole Plant Solanum lycopersicum" by Dhruv Aditya Srivastava, Gulab Chand Arya, Eswari PJ Pandaranayaka, Ekaterina Manasherova, Dov B. Prusky, Yigal Elad, Omer Frenkel, and Arye Harel, the Short Read Archive accession numbers have been replaced with the NCBI BioProject number and the BioProject URL has been added as an Author-Recommended Internet Resource. None of the conclusions of the paper have been altered. 